\title{
Síndrome da fragilidade biológica em idosos: revisão sistemática
}

\section{Biological fragility syndrome in the elderly: systematic review}

\author{
Sheilla Tribess ${ }^{1}$ e Ricardo Jacó de Oliveira ${ }^{2}$
}

1 Instituto de Ciências da Saúde, Universidade Federal do Triângulo Mineiro. Uberaba, MG, Brasil. sheilla@ef.uftm.edu.br

2 Faculdade de Educação Física, Universidade de Brasília. Brasília, DF, Brasil. rjaco@unb.br

Recebido 27 Novembro 2010/Enviado para Modificação 3 Outubro 2011/Aprovado 23 Outubro 2011

\section{RESUMO}

Objetivo O objetivo desse estudo foi identificar a prevalência e as estratégias de avaliação da Síndrome da Fragilidade Biológica em Idosos.

Metodologia Para o desenvolvimento deste estudo foi realizada uma busca em base de dados eletrônica (Medline/Pubmed) e lista de referências dos artigos identificados, utilizando os seguintes descritores, na língua inglesa: "frailty e "frail" conjugados com os termos "elderly", "aging" e "prevalence". Estes termos/descritores foram combinados usando os operadores lógicos disponíveis nas ferramentas de buscas. A busca eletrônica inicial resultou em 1865 manuscritos. O processo de análise dos estudos envolveu leitura de títulos, resumos e textos completos. Após todas essas fases, 35 manuscritos preencheram os critérios de inclusão da revisão. Resultados Os resultados indicaram que as mulheres, com índices que variam de $7,3 \%$ a $21,6 \%$, são mais frágeis do que os homens, com percentuais que variam de $4 \%$ a $19,2 \%$.

Conclusão As disparidades na prevalência de índices de fragilidade e pré-fragilidade devem ser minimizadas, com estímulo a padronização metodológica para a avaliação da fragilidade humana.

Palavras-chave: Idoso fragilizado, prevalência, revisão (fonte: DeCS, BIREME).

\section{ABSTRACT}

Objective The aim of this study was to identify the prevalence and assessment strategies of Biological Fragility Syndrome in the Elderly.

Methods For the development of this study was it was done a search in electronic databases (Medline/PubMed) and the reference lists of articles identified using the following key words/terms in English: "frailty" and 'frail" in conjunction with the terms "elderly", "aging" and "prevalence". These terms/descriptors were combined using the logical operators available in search engines. The initial electronic search resulted in 1865 manuscripts. The process of analysis of the studies involved reading titles, abstracts and full texts. After all these phases, 35 manuscripts met the inclusion criteria of the review. 
Results The results indicated that women, with rage from $7.3 \%$ to $21.6 \%$, are frailer than men, with percentages ranging from $4 \%$ to $19.2 \%$.

Conclusions Differences in prevalence rates of prefrailty and frailty should be minimized, with stimulus for standardization for the evaluation of human frailty.

Key Words: Frail elderly, prevalence, review (source: MeSH, NLM).

\section{RESUMEN}

Síndrome de fragilidad biológica en el anciano: revisión sistemática

Objetivo El objetivo del estudio fue identificar las estrategias y la evaluación de la prevalencia de síndrome de fragilidad biológica en el anciano.

Metodología Para desarrollar este estudio se llevó a cabo una búsqueda en bases de datos electrónicas (Medline/Pubmed) y listas de referencias de los artículos identificados con los siguientes descriptores en Inglés: "fragilidad" y "frágil", en relación con los términos "personas mayores", "envejecimiento" y "prevalencia". Estos términos o descriptores se combinaron mediante los operadores lógicos disponibles en los motores de búsqueda. El resultado de la búsqueda electrónica resultó en 1865 manuscritos. El proceso de análisis de los estudios incluyó la lectura de los títulos, resúmenes y textos completos. Después de todas estas fases, 35 manuscritos cumplieron los criterios de inclusión de la revisión.

Resultados Los resultados indicaron que las mujeres, con tasas que van del $7,3 \%$ al $21,6 \%$, son más frágiles que los hombres, con porcentajes que van del $4 \%$ al $19,2 \%$.

Conclusión Las diferencias en las tasas de prevalencia de pre-fragilidad y la fragilidad deben reducirse al mínimo, con la normalización de estímulo para la evaluación de la fragilidad humana.

Palabras Clave: Anciano frágil, prevalencia, revisión (fuente: DeCS, BIREME).

$\mathrm{O}$ aumento da população idosa aumentou o interesse de diversas áreas do conhecimento, em estudar meios que favoreçam as pessoas com mais idade em obter uma vida com qualidade. Na área da saúde os estudos se intensificam na manutenção da independência funcional, nesse sentido o entendimento do termo frágil passa a ser necessário para a formulação de intervenções efetivas.

Apesar de não se ter uma definição concisa do que venha ser caracterizado o estado de fragilidade na pessoa idosa, há balizadores comuns que indicam que esse conceito é amplo e dinâmico envolvendo aspectos tanto biomédicos como psicossociais (1-4). De um modo geral, é possível afirmar que o estado frágil de uma pessoa idosa é um processo prolongado de incapacidade que indica vulnerabilidade, predisposição ao declínio funcional e no estágio mais avançado a morte $(4,5)$. 
A dificuldade de se obter um consenso na caracterização da fragilidade humana resulta no estabelecimento de barreiras na comunicação entre os especialistas da área. Pois, os estudos realizados invibializam as generalizações, em virtude de diferentes instrumentos e critérios utilizados para avaliar a síndrome da fragilidade em pessoas idosas.

O propósito desse estudo foi identificar a prevalência da síndrome de fragilidade e os respectivos critérios de avaliação. Com o entendimento que a síndrome da fragilidade é uma complexa interação de fatores biopsicossociais e que a coletânea dos instrumentos utilizados na identificação do estado frágil possibilitará indicar os balizadores mais utilizados na avaliação.

\section{MÉTODOS}

A revisão sistemática da literatura foi realizada a partir da busca por artigos originais sobre a síndrome de fragilidade em idosos, em base de dados eletrônica (Medline/Pubmed) e lista de referências dos artigos identificados durante os meses de julho e agosto de 2009. As referências que preencheram os critérios de inclusão foram avaliadas, independentemente do periódico. A seleção dos descritores utilizados no processo de revisão foi efetuada mediante consulta ao Medical Subject Headings (MeSH).

Nas buscas, os seguintes descritores, em língua inglesa, foram considerados: "frailty e "frail" conjugados com os termos "elderly", "aging" e/ou "prevalence". Recorreu-se aos operadores lógicos "AND", "OR" e "AND NOT" para combinação dos descritores e termos utilizados para rastreamento das publicações.

Através deste procedimento de busca, foram identificadas, inicialmente, 1 865 publicações potencialmente elegíveis para inclusão nesta revisão.

Em seguida, identificaram-se os artigos que atenderam aos seguintes critérios de inclusão: a. A mostra deveria incluir idosos mesmo que abrangendo outras faixas de idade, desde que categorizado por faixas etárias; b. A amostra não poderia incluir idosos hospitalizados e ou sobre efeito de medicamentos; c. Artigo original de pesquisa com seres humanos; d. Foram excluídos artigos de revisão, assim como, publicações em forma de carta ou conferência; e. Foram incluídos somente os artigos em língua inglesa, espanhola e portuguesa; e, f. Publicação até agosto de 2009. Optou-se por não incluir teses, dissertações e monografias, visto que a realização de uma busca sistemática das mesmas é inviável logisticamente. 
Após a primeira análise, com avaliação dos títulos e resumos, 109 artigos foram considerados elegíveis para a segunda fase desta revisão, que consistiu da leitura dos artigos na íntegra. Ao final, apenas 35 artigos atenderam a todos os critérios de inclusão.

$\mathrm{Na}$ avaliação dos artigos, foram observados os seguintes aspectos:

- amostra (dimensionamento adequado, envolvendo seleção aleatória de participantes e representatividade);

- instrumentos e indicadores utilizados para avaliar a fragilidade em idosos;

- definição operacional do termo Fragilidade;

- prevalência da fragilidade em idosos.

\section{RESULTADOS}

Na Tabela 1, são apresentadas informações gerais sobre os 35 estudos incluídos na revisão que abrangeram o período de 2000 a 2009. Destes, 27 foram publicados nos últimos cinco anos (entre 2005 a 2009), indicando o crescente interesse pelo assunto da fragilidade em idosos.

Dos artigos analisados 24 foram realizados na América do Norte, sendo 17 nos Estados Unidos da América e sete no Canadá; seis nos países europeus (Inglaterra, Itália, Holanda e França), quatro na China e um na Oceania (Austrália).

Uma das publicações (6) traz em seu corpo do texto informações sobre dois levantamentos realizados em períodos distintos (1995/1996 e 2006), todavia, os resultados dessas duas coletas de dados foram incluídos nas tabelas de forma separada tendo a mesma referência como base.

Dos estudos analisados, três compararam diferentes índices para avaliar a fragilidade. Ensrud et al. (7) e Bandeen-Roche et al. (8) analisaram dois índices entre si e Cigolle et al. (9) comparou os resultados de três índices de fragilidade. Os resultados foram apresentados separadamente, identificando o índice de fragilidade adotado e a referência de base.

Também se verificou que dois estudos compararam a fragilidade em duas populações distintas, americanas mexicanas e americanas européias (10); e americanos africanos e americanos brancos (11). Ambos os estudos foram apresentados de forma discriminada tendo a mesma referência como base. 
A composição das amostras variou em relação à representatividade em que alguns eram correspondentes a uma determinada região, país ou representativo ao continente europeu (12). Em apenas três estudos os critérios de representatividade da amostra não foram identificados e a forma de seleção das pessoas do estudo foi por conveniência (13-15).

Tabela 1. Descrição dos artigos inclusos na revisão

\begin{tabular}{|c|c|c|c|c|c|}
\hline Autor & Ano & Periódico & Idade & $\mathrm{n}$ & Local \\
\hline Brown (13) & 2000 & J Gerontol A Biol Sci Med Sci & $>77$ & 107 & EUA \\
\hline Fried. (1) & 2001 & J Gerontol A Biol Sci Med Sci & $\geq 65$ & 5317 & EUA \\
\hline Mitnitski (16) & 2002 & BMC Geriatr & $\geq 65$ & 2914 & Canadá \\
\hline Kirby (15) & 2004 & $\begin{array}{l}\text { J Gerontol B Psychol Sci Soc } \\
\text { Sci }\end{array}$ & $65-95$ & 233 & Inglaterra \\
\hline Mitnitski.(19) & 2004 & J Gerontol A Biol Sci Med Sci & $\geq 65$ & 9008 & Canadá \\
\hline Villareal (14) & 2004 & Obes Res & $\geq 65$ & 156 & EUA \\
\hline Songl.(20) & 2004 & J Am Geriatr Soc & $65-99$ & 9008 & Canadá \\
\hline Rockwood.(24) & 2004 & J Gerontol A Biol Sci Med Sci & $\geq 65$ & 9008 & Canadá \\
\hline Blaum (26) & 2005 & J Am Geriatr Soc & $70-79$ & 595 & EUA \\
\hline Ottenbacher.(27) & 2005 & J Am Geriatr Soc & $\geq 70$ & 621 & EUA \\
\hline Gogginsl.(22) & 2005 & J Gerontol A Biol Sci Med Sci & $\geq 70$ & 2032 & China \\
\hline Klein.(5) & 2005 & Arc Gerontol Geriatric & $43-86$ & 2515 & EUA \\
\hline Puts (25) & 2005 & J Am Geriatr Soc & $\geq 65$ & 2257 & Holanda \\
\hline Woods.(28) & 2005 & J Am Geriatr Soc & 65 a 79 & 4657 & EUA \\
\hline Bandeen-R. (8) & 2006 & J Gerontol A Biol Sci Med Sci & $\geq 65$ & 1438 & EUA \\
\hline Woo.(23) & 2005 & Gerontology & $\geq 70$ & 2032 & China \\
\hline Hirsch.(11) & 2006 & Ann Epidemiol & $\geq 65$ & 5277 & EUA \\
\hline Bartali.(29) & 2006 & J Gerontol A Biol Sci Med Sci & $65-93$ & 802 & Itália \\
\hline Cesari.(30) & 2006 & Am J Clin Nutr & $65-102$ & 923 & Itália \\
\hline Song.(21) & 2007 & Aging Clin Exp Res & $\geq 65$ & 8547 & Canadá \\
\hline Cawthon.(31) & 2007 & J Am Geriatr Soc & $\geq 65$ & 5993 & EUA \\
\hline Ensrud (3) & 2007 & J Gerontol A Biol Sci Med Sci & $\geq 69$ & 6724 & EUA \\
\hline Rockwood (2) & 2007 & J Gerontol A Biol Sci Med Sci & $\geq 70$ & 2305 & Canadá \\
\hline Andrew (32) & 2008 & Plos One & $\geq 65$ & 3707 & Canadá \\
\hline Ensrud.(7) & 2008 & Arch Intern Med & $\geq 69$ & 6701 & EUA \\
\hline Espinoza (10) & 2008 & J Am Geriatr Soc & $65-80$ & 749 & EUA \\
\hline Avila-Funesl (33) & 2008 & J Gerontol A Biol Sci Med Sci & $\geq 65$ & 6078 & França \\
\hline Blyth (34) & 2008 & Pain & $\geq 70$ & 1705 & Austrália \\
\hline Santos-E. (12) & 2009 & J Gerontol A Biol Sci Med Sci & $\geq 50$ & 16584 & Europa \\
\hline Ottenbacher (6) & 2009 & Am J Public Health & $\geq 65$ & 2049 & EUA \\
\hline $\mathrm{Gu}(17)$ & 2009 & $\begin{array}{l}\text { J Gerontol B Psychol Sci Soc } \\
\text { Sci }\end{array}$ & 65-109 & 15919 & China \\
\hline Masel (35) & 2009 & Health Qual Life Outcomes & $\geq 74$ & 1008 & EUA \\
\hline Cigolle.(9) & 2009 & J Am Geriatr Soc & $\geq 65$ & 11113 & EUA \\
\hline Dupre (18) & 2009 & BMJ & $65-109$ & 13717 & China \\
\hline Buchman.(4) & 2009 & Exp Aging Res & 80,4 & 832 & EUA \\
\hline
\end{tabular}

Todos os estudos descritos na revisão abordavam a população na faixa etária igual ou acima de 65 anos, com exceção de dois estudos no qual abordavam a faixa etária de 43 a 86 anos (5) e acima de 50 anos (12), que foram incluídos na revisão por discriminar os resultados por faixa etária, sendo assim, utilizou-se apenas os resultados da amostra com 65 anos ou mais. 
A Tabela 2 ressalta a prevalência de fragilidade em idosos, para tanto foram aceitos os estudos que destacaram as seguintes variações na definição operacional das categorias da fragilidade (frágil e pré-frágil).

Tabela 2. Prevalência da síndrome biológica de fragilidade em idosos

\begin{tabular}{|c|c|c|c|c|c|c|}
\hline \multirow[b]{2}{*}{ Autor } & \multicolumn{2}{|c|}{ Homens } & \multicolumn{2}{|c|}{ Mulheres } & \multicolumn{2}{|c|}{ Geral } \\
\hline & $\begin{array}{c}\text { frágil } \\
\%\end{array}$ & $\begin{array}{c}\text { préfragil } \\
\%\end{array}$ & $\begin{array}{c}\text { frágil } \\
\%\end{array}$ & $\begin{array}{c}\text { préfragil } \\
\%\end{array}$ & $\begin{array}{c}\text { fragil } \\
\%\end{array}$ & $\begin{array}{c}\text { préfrágil } \\
\%\end{array}$ \\
\hline Fried et al.(1) & 4,9 & - & 7,3 & - & 6,9 & - \\
\hline Blaum et al.(26) & & & & & 7,0 & 43,1 \\
\hline Ottenbacher et al.(27) & 17 & 33 & 22 & 33 & 20 & 33 \\
\hline Woods et al.(28) & - & - & 16,3 & 28,3 & - & - \\
\hline Bandeen-Roche et al.(8) & - & - & $11,6^{\mathrm{a}}$ & $55,2^{a}$ & - & - \\
\hline Bandeen-Roche et al.(8) & - & - & $11,3^{b}$ & $43,8^{b}$ & - & - \\
\hline Hirsch et al.(11) & $8,7^{\mathrm{c}}$ & - & $15,0^{c}$ & - & - & - \\
\hline Hirsch et al.(11) & $4,6^{d}$ & - & $6,8^{d}$ & - & - & - \\
\hline Cesari et al.(30) & & & & & 8,8 & - \\
\hline Cawthon et al.(31) & 4,0 & 40,0 & - & - & - & - \\
\hline Ensrud et al.(3) & - & - & 16,3 & 47,0 & & \\
\hline Rockwood et al.(2) & - & - & - & - & 16,5 & 35,7 \\
\hline Ensrud et al.(7) & - & - & $17,1^{\mathrm{e}}$ & $36,4^{e}$ & - & - \\
\hline Ensrud et al.(7) & - & - & $16,2^{\mathrm{a}}$ & $47^{a}$ & - & - \\
\hline Espinoza e Hazuda (10) & - & - & - & - & $11,3^{\dagger}$ & - \\
\hline Espinoza e Hazuda (10) & - & - & - & - & $7,0^{\mathrm{g}}$ & - \\
\hline Avila-Funes et al.(33) & 7,0 & 47,7 & 8,7 & 49,3 & 4,3 & 45,1 \\
\hline Blyth et al.(34) & 9,4 & 40,6 & - & - & - & - \\
\hline Santos-Eggiman et al.(12) & 11,9 & 41,9 & 21.0 & 42,7 & 17.0 & 42,3 \\
\hline Ottenbacher et al.(6) & $8,5^{\mathrm{h}}$ & $46,2^{h}$ & $6,9^{\text {h }}$ & $48,5^{\mathrm{h}}$ & $7,0^{\mathrm{n}}$ & $48,0^{\mathrm{h}}$ \\
\hline Ottenbacher et al.(6) & 19,2 & 55,6 & $21,6^{\prime}$ & $54,1^{\prime}$ & $21^{\prime}$ & $55^{i}$ \\
\hline Masel et al.(35) & - & - & - & - & $20^{\dagger}$ & $54^{\dagger}$ \\
\hline Cigolle et al.(9) & - & - & - & - & $20,3^{j}$ & - \\
\hline Cigolle et al.(9) & - & - & - & - & 15,4 & - \\
\hline Cigolle et al.(9) & - & - & - & - & $10,9^{\mathrm{m}}$ & - \\
\hline
\end{tabular}

Os 18 estudos analisados indicaram uma ampla variação de prevalência de fragilidade, com uma variação geral de 6,9 a 21,0 \% para o estado frágil e 33 a $55 \%$ para o estado pré-frágil. Quando separado por sexo, evidenciouse para os homens uma fragilidade de 4 a $19,2 \%$ e 33 a $55,6 \%$ de estado pré-frágil, já para as mulheres, 6,8 a $22 \%$ de idosas frágeis e 23,3 a $54,1 \%$ de idosas pré-frágeis.

No Quadro 1, estão apresentados as especificações do instrumento utilizado, indicadores analisados e a definição da variável utilizada nos estudos.

Entre os instrumentos utilizados, o Fenótipo da Fragilidade do CHS (Cardiovascular Health Study) ou modificações dele foi citado em 20 estudos; 
há ainda o Índice de Fragilidade que utiliza 20 indicadores (16), 39 indicadores $(17,18), 40$ indicadores (19-21), 62 indicadores $(22,23), 70$ indicadores (2); o Teste de Performance Física $(13,14)$; a Escala Geriátrica (24); Marcos de Fragilidade (5,25); índice do estudo de osteoporose fraturas (7); Modelo de deficiência e Modelo de Burden (9) e Composição da Fragilidade (4).

Quadro 1. Análises dos artigos segundo os instrumentos, definição operacional das variáveis e nível de exposição

\begin{tabular}{|c|c|c|}
\hline $\begin{array}{l}\text { Referência e Especificação do } \\
\text { Instrumento }\end{array}$ & Indicadores Analisados & $\begin{array}{l}\text { Definição } \\
\text { Operacional da } \\
\text { Variável }\end{array}$ \\
\hline $\begin{array}{l}\text { Brown et al.(13) } \\
\text { Teste de Performance Física }\end{array}$ & $\begin{array}{l}\text { Força, amplitude de movimento, } \\
\text { equilíbrio, coordenação, velocidade de } \\
\text { reação, análise da marcha e } \\
\text { sensibilidade. }\end{array}$ & $\begin{array}{l}\text { não frágil, leve a } \\
\text { moderada } \\
\text { fragilidade }\end{array}$ \\
\hline $\begin{array}{l}\text { Fried et al. (1) } \\
\text { Fenótipo da Fragilidade CHS }\end{array}$ & $\begin{array}{l}\text { Perda de peso não intencional, } \\
\text { exaustão, fraqueza, velocidade de } \\
\text { marcha e baixa atividade física. }\end{array}$ & $\begin{array}{l}\text { frágil, pré-frágil e } \\
\text { não frágil }\end{array}$ \\
\hline $\begin{array}{l}\text { Mitnitski et al. (16) } \\
\text { Índice de Fragilidade (20 } \\
\text { indicadores) }\end{array}$ & $\begin{array}{l}\text { Perda de atividades funcionais, } \\
\text { sensoriais, e problemas médicos, de } \\
\text { saúde e de comportamento. }\end{array}$ & frágil e apto \\
\hline $\begin{array}{l}\text { Kirby et al.(15) } \\
\text { Fragilidade de Strawbridge }\end{array}$ & $\begin{array}{l}\text { Função física, nutricional e cognitiva e } \\
\text { problemas sensoriais. }\end{array}$ & frágil e não frágil \\
\hline $\begin{array}{l}\text { Mitnitski et al.(19) } \\
\text { Índice de Fragilidade (40 } \\
\text { indicadores) }\end{array}$ & $\begin{array}{l}\text { Sintomas, atitudes, doenças e } \\
\text { funcionalidade. }\end{array}$ & frágil e apto \\
\hline $\begin{array}{l}\text { Villareal et al. (14) } \\
\text { Teste de Performance Física }\end{array}$ & $\begin{array}{l}\text { Função motora, } \mathrm{VO}_{2} \text {, atividades da } \\
\text { vida diária. }\end{array}$ & $\begin{array}{l}\text { não frágil e leve a } \\
\text { moderada } \\
\text { fragilidade }\end{array}$ \\
\hline $\begin{array}{l}\text { Song et al.(20) } \\
\text { Indice de Fragilidade ( } 40 \\
\text { indicadores) }\end{array}$ & $\begin{array}{l}\text { Sintomas, atitudes, doenças e } \\
\text { funcionalidade. }\end{array}$ & $\begin{array}{l}\text { fragilidade leve a } \\
\text { moderada }\end{array}$ \\
\hline $\begin{array}{l}\text { Rockwood et al.(24) } \\
\text { Geriatric Status Scale }\end{array}$ & Performance cognitiva e funcional. & $\begin{array}{l}\text { fragilidade leve, } \\
\text { moderada e } \\
\text { severa }\end{array}$ \\
\hline $\begin{array}{l}\text { Blaum et al.(26) } \\
\text { Fenótipo da Fragilidade } \mathrm{CHS}\end{array}$ & $\begin{array}{l}\text { Perda de peso não intencional, } \\
\text { exaustão, fraqueza, velocidade de } \\
\text { marcha e baixa atividade física. }\end{array}$ & $\begin{array}{l}\text { frágil, pré-frágil e } \\
\text { não frágil }\end{array}$ \\
\hline $\begin{array}{l}\text { Ottenbacher et al.(27) } \\
\text { Fenótipo da fragilidade CHS e } \\
\text { Índice da Fragilidade de } \\
\text { Walston Adaptado }\end{array}$ & $\begin{array}{l}\text { Perda de peso não intencional, } \\
\text { exaustão, velocidade de marcha e } \\
\text { força de grip. }\end{array}$ & $\begin{array}{l}\text { frágil, pré-frágil e } \\
\text { não frágil }\end{array}$ \\
\hline $\begin{array}{l}\text { Goggins et al.(22) } \\
\text { Índice de Fragilidade ( } 62 \\
\text { variáveis) }\end{array}$ & $\begin{array}{l}\text { Estado de saúde social, funcional, } \\
\text { físico e mental. }\end{array}$ & apto e frágil \\
\hline $\begin{array}{l}\text { Klein et al.(5) } \\
\text { Marcos de fragilidade Adaptado }\end{array}$ & $\begin{array}{l}\text { Velocidade de caminhada, força } \\
\text { manual, pico de fluxo expiratório, } \\
\text { levantar da cadeira sem ajuda das } \\
\text { mãos e acuidade visual. }\end{array}$ & $\begin{array}{l}\text { não frágil e leve, } \\
\text { moderada e } \\
\text { severa fragilidade. }\end{array}$ \\
\hline $\begin{array}{l}\text { Puts et al.(25) } \\
\text { Marcos de fragilidade }\end{array}$ & $\begin{array}{l}\text { Peso corporal, pico de fluxo } \\
\text { expiratório, problemas cognitivos, de } \\
\text { visão e audição, incontinência urinária, } \\
\text { sintomas depressivos e atividade } \\
\text { física. }\end{array}$ & frágil e não frágil \\
\hline $\begin{array}{l}\text { Woods et al.(28) } \\
\text { Fenótipo da Fragilidade CHS }\end{array}$ & $\begin{array}{l}\text { Perda de peso não intencional, } \\
\text { exaustão, fraqueza, velocidade de } \\
\text { marcha e baixa atividade física. }\end{array}$ & $\begin{array}{l}\text { frágil, pré-frágil e } \\
\text { não frágil }\end{array}$ \\
\hline $\begin{array}{l}\text { Woo et al.(23) } \\
\text { Índice de Fragilidade ( } 62 \\
\text { variáveis) }\end{array}$ & Saúde cognitiva, psicológica e física. & $\begin{array}{l}\text { fragilidade num } \\
\text { Intervalo de } 0-1\end{array}$ \\
\hline
\end{tabular}




\begin{tabular}{|c|c|c|}
\hline $\begin{array}{l}\text { Referência e Especificação do } \\
\text { Instrumento }\end{array}$ & Indicadores Analisados & $\begin{array}{l}\text { Definição } \\
\text { Operacional da } \\
\text { Variável }\end{array}$ \\
\hline $\begin{array}{l}\text { Bandeen-Roche et al.(8) } \\
\text { Fenótipo da Fragilidade CHS }\end{array}$ & $\begin{array}{l}\text { Perda de peso não intencional, } \\
\text { exaustão, fraqueza, velocidade de } \\
\text { marcha e baixa atividade física }\end{array}$ & $\begin{array}{l}\text { frágil, pré-frágil e } \\
\text { não frágil }\end{array}$ \\
\hline $\begin{array}{l}\text { Women's Health and Aging } \\
\text { Studies }\end{array}$ & $\begin{array}{l}\text { Perda de peso não intencional, } \\
\text { exaustão, fraqueza, velocidade de } \\
\text { marcha e baixa atividade física. }\end{array}$ & $\begin{array}{l}\text { frágil, pré-frágil e } \\
\text { não frágil }\end{array}$ \\
\hline $\begin{array}{l}\text { Hirsch et al.(11) } \\
\text { Fenótipo da Fragilidade CHS }\end{array}$ & $\begin{array}{l}\text { Perda de peso não intencional, } \\
\text { exaustão, fraqueza, velocidade de } \\
\text { marcha e baixa atividade física. }\end{array}$ & $\begin{array}{l}\text { frágil, pré-frágil e } \\
\text { não frágil }\end{array}$ \\
\hline $\begin{array}{l}\text { Bartali et al.(29) } \\
\text { Fenótipo da fragilidade CHS } \\
\text { adaptado }\end{array}$ & $\begin{array}{l}\text { Exaustão, fraqueza, velocidade de } \\
\text { marcha e baixa atividade física. }\end{array}$ & frágil e não frágil \\
\hline $\begin{array}{l}\text { Cesari et al.(30) } \\
\text { Fenótipo da Fragilidade CHS }\end{array}$ & $\begin{array}{l}\text { Perda de peso não intencional, } \\
\text { exaustão, fraqueza, velocidade de } \\
\text { marcha e baixa atividade física. }\end{array}$ & frágil, pré-frágil \\
\hline $\begin{array}{l}\text { Song et al.(21) } \\
\text { Îndice de Fragilidade (40 } \\
\text { indicadores) }\end{array}$ & $\begin{array}{l}\text { Déficits de saúde (sintomas, doenças, } \\
\text { incapacidades, condições } \\
\text { desfavoráveis de vida). }\end{array}$ & $\begin{array}{l}\text { fragilidade leve a } \\
\text { moderada }\end{array}$ \\
\hline $\begin{array}{l}\text { Cawthon et al.(31) } \\
\text { Fenótipo da fragilidade CHS } \\
\text { adaptado }\end{array}$ & $\begin{array}{l}\text { Sarcopenia, fraqueza, exaustão, baixa } \\
\text { atividade física e velocidade de } \\
\text { marcha. }\end{array}$ & $\begin{array}{l}\text { frágil, pré-frágil e } \\
\text { robusto. }\end{array}$ \\
\hline $\begin{array}{l}\text { Ensrud et al.(3) } \\
\text { Fenótipo da Fragilidade CHS }\end{array}$ & $\begin{array}{l}\text { Perda de peso não intencional, } \\
\text { exaustão, fraqueza, velocidade de } \\
\text { marcha e baixa atividade física. }\end{array}$ & $\begin{array}{l}\text { frágil, pré-frágil e } \\
\text { robusto. }\end{array}$ \\
\hline $\begin{array}{l}\text { Rockwood et al.(2) } \\
\text { Fenótipo da Fragilidade CHS }\end{array}$ & $\begin{array}{l}\text { Perda de peso não intencional, } \\
\text { exaustão, fraqueza, velocidade de } \\
\text { marcha e baixa atividade física. }\end{array}$ & $\begin{array}{l}\text { frágil, pré-frágil e } \\
\text { robusto }\end{array}$ \\
\hline $\begin{array}{l}\text { Índice de Fragilidade (70 } \\
\text { indicadores) }\end{array}$ & $\begin{array}{l}\text { Doenças, atividade da vida diária, } \\
\text { sinais físicos e neurológicos. }\end{array}$ & $\begin{array}{l}\text { fragilidade num } \\
\text { escore de } 0-1\end{array}$ \\
\hline $\begin{array}{l}\text { Andrew et al.(32) } \\
\text { Índice de Fragilidade }\end{array}$ & $\begin{array}{l}\text { Perda de atividades funcionais, } \\
\text { sensoriais, e problemas médicos, de } \\
\text { saúde e de comportamento. }\end{array}$ & $\begin{array}{l}\text { fragilidade num } \\
\text { intervalo de } 0-1 .\end{array}$ \\
\hline $\begin{array}{l}\text { Ensrud et al.(7) } \\
\text { Indice SOF (Study of } \\
\text { Osteoporotic Fractures) }\end{array}$ & $\begin{array}{l}\text { Perda de peso, incapacidade de } \\
\text { levantar da cadeira } 5 \text { vezes seguida e } \\
\text { falta de energia. }\end{array}$ & $\begin{array}{l}\text { frágil, pré-frágil e } \\
\text { robusto. }\end{array}$ \\
\hline Fenótipo da Fragilidade CHS & $\begin{array}{l}\text { Perda de peso não intencional, } \\
\text { exaustão, fraqueza, velocidade de } \\
\text { marcha e baixa atividade física. }\end{array}$ & $\begin{array}{l}\text { frágil, pré-frágil e } \\
\text { robusto }\end{array}$ \\
\hline $\begin{array}{l}\text { Espinoza e Hazuda (10) } \\
\text { Fenótipo da Fragilidade CHS }\end{array}$ & $\begin{array}{l}\text { Perda de peso não intencional, } \\
\text { exaustão, fraqueza, velocidade de } \\
\text { marcha e baixa atividade física. }\end{array}$ & frágil, pré-frágil \\
\hline $\begin{array}{l}\text { Avila-Funes et al.(33) } \\
\text { Fenótipo da fragilidade CHS } \\
\text { adaptado }\end{array}$ & $\begin{array}{l}\text { Perda de peso, fraqueza, baixa } \\
\text { resistência e energia, lentidão e baixa } \\
\text { atividade física. }\end{array}$ & $\begin{array}{l}\text { frágil, pré-frágil e } \\
\text { não frágil }\end{array}$ \\
\hline $\begin{array}{l}\text { Blyth et al.(34) } \\
\text { Fenótipo da Fragilidade CHS }\end{array}$ & $\begin{array}{l}\text { Perda de peso não intencional, } \\
\text { exaustão, fraqueza, velocidade de } \\
\text { marcha e baixa atividade física. }\end{array}$ & $\begin{array}{l}\text { frágil, pré-frágil e } \\
\text { não frágil }\end{array}$ \\
\hline $\begin{array}{l}\text { Santos-Eggiman et al.(12) } \\
\text { Fenótipo da Fragilidade CHS }\end{array}$ & $\begin{array}{l}\text { Exaustão, encolhimento, fraqueza, } \\
\text { lentidão e baixa atividade física. }\end{array}$ & $\begin{array}{l}\text { frágil, pré-frágil e } \\
\text { não frágil }\end{array}$ \\
\hline $\begin{array}{l}\text { Ottenbacher et al.(6) } \\
\text { Fenótipo da Fragilidade } \mathrm{CHS}\end{array}$ & $\begin{array}{l}\text { Perda de peso não intencional, } \\
\text { exaustão, fraqueza, velocidade de } \\
\text { marcha e atividade física. }\end{array}$ & $\begin{array}{l}\text { frágil, pré-frágil e } \\
\text { não frágil }\end{array}$ \\
\hline
\end{tabular}




\begin{tabular}{|c|c|c|}
\hline $\begin{array}{l}\text { Referência e Especificação do } \\
\text { Instrumento }\end{array}$ & Indicadores Analisados & $\begin{array}{c}\text { Definição } \\
\text { Operacional da } \\
\text { Variável }\end{array}$ \\
\hline $\begin{array}{l}\text { Gu et al.(17) } \\
\text { Índice de Fragilidade ( } 39 \\
\text { indicadores) }\end{array}$ & $\begin{array}{l}\text { Estado de saúde referido, função } \\
\text { cognitiva, depressão, habilidade, } \\
\text { visual e auditiva, incapacidade, } \\
\text { doenças e ritmo cardíaco. }\end{array}$ & $\begin{array}{l}\text { fragilidade num } \\
\text { Intervalo de } 0-1 \text { e } \\
\text { categorizada em } \\
\text { quartis. }\end{array}$ \\
\hline $\begin{array}{l}\text { Masel et al.(35) } \\
\text { Fenótipo da Fragilidade CHS }\end{array}$ & $\begin{array}{l}\text { Perda de peso não intencional, } \\
\text { exaustão, fraqueza, velocidade de } \\
\text { marcha e baixa atividade física. }\end{array}$ & $\begin{array}{l}\text { frágil, pré-frágil e } \\
\text { não frágil }\end{array}$ \\
\hline $\begin{array}{l}\text { Cigolle et al.(9) } \\
\text { Índice de Fragilidade }\end{array}$ & $\begin{array}{l}\text { Função física, nutricional, cognitiva e } \\
\text { problemas sensórios. }\end{array}$ & $\begin{array}{l}\text { frágil } \geq 2 \text { domínios } \\
\text { com deficiência }\end{array}$ \\
\hline $\begin{array}{l}\text { Modelo de Burden Canadian } \\
\text { Study of Family Medicine }\end{array}$ & $\begin{array}{l}\text { Doenças, condições neurológicas ou } \\
\text { prejuizos na cognição, humor, } \\
\text { mobilidade, ou função. }\end{array}$ & $\begin{array}{l}\text { frágil }(\geq 0,2) \text { e não } \\
\text { frágil }\end{array}$ \\
\hline Fenótipo da Fragilidade CHS & $\begin{array}{l}\text { Perda de peso, exaustão, baixa } \\
\text { atividade física, velocidade de marcha } \\
\text { e fraqueza. }\end{array}$ & frágil e não frágil \\
\hline $\begin{array}{l}\text { Dupre et al.(18) } \\
\text { Índice de Fragilidade ( } 39 \\
\text { indicadores) }\end{array}$ & $\begin{array}{l}\text { Estado de saúde referido, função } \\
\text { cognitiva, habilidade visual e auditiva, } \\
\text { depressão, incapacidade, doenças e } \\
\text { ritmo cardíaco. }\end{array}$ & $\begin{array}{l}\text { fragilidade num } \\
\text { Intervalo de } 0-1 \text { e } \\
\text { categorizada em } \\
\text { quartis. }\end{array}$ \\
\hline $\begin{array}{l}\text { Buchman et al.(4) } \\
\text { Composição de fragilidade }\end{array}$ & $\begin{array}{l}\text { Força de grip, fadiga, velocidade de } \\
\text { marcha e composição corporal. }\end{array}$ & $\begin{array}{l}\text { menos frágil a } \\
\text { mais frágil }\end{array}$ \\
\hline
\end{tabular}

\section{DISCUSSÃO}

O aumento da proporção de idosos na população mundial faz com que os gestores públicos aumentem sua atenção e destinem investimentos que garantam condições de vida com qualidade para essas pessoas na velhice. Nesse sentido, a avaliação da prevalência de fragilidade é fundamental para o desenvolvimento, implantação e avaliação de políticas públicas na promoção de saúde para a população idosa.

O crescente aumento de estudos nos últimos cinco anos, principalmente na América do Norte, enfatizam a importância do diagnóstico de fragilidade na população idosa. No entanto, as dificuldades de se estabelecer um conceito comum sobre o termo fragilidade proporcionam caminhos diversificados para o seu diagnóstico. Vários instrumentos foram desenvolvidos para operacionalizar a construção da fragilidade, inclusive índices recentes com base no julgamento clínico, performance física, acumulação de déficits e avaliação geriátrica. Muito embora, verificou-se uma prevalência nos artigos analisado do instrumento Fenótipo da Fragilidade, desenvolvido pelo Cardiovascular Health Study (CHS), e suas variações.

A validade do índice CHS em predizer risco de resultados adversos, incluindo comprometimento funcional, quedas, hospitalização, fratura e morte foi confirmada em estudos com grupos de adultos mais velhos $(3,8,28,31)$. Além disso, o índice CHS tem sido associado a alterações específicas em fatores fisiológicos 
fornecendo evidências de comprometimento dos múltiplos sistemas biológicos e moleculares que podem estar subjacentes ao desenvolvimento da síndrome da fragilidade clínica (1). A abordagem fenotípica define fragilidade em três níveis: robusto/não frágil, pré-frágil e frágil, com base em indicadores, tais como perda de peso, cansaço/exaustão, fraqueza, lentidão na marcha e atividade física reduzida.

Não há um padrão ouro para avaliar a fragilidade humana, o que se verificou nos trabalhos são instrumentos que utilizam parâmetros diversificados, tanto de desempenho como de percepção, analisados separados ou conjuntamente. Essa diversidade proporciona divergências metodológicas, seja na escolha do instrumento e na definição do ponto de corte para a exposição à fragilidade, que comprometem a comparação dos estudos.

Isso pode ser visualizado nos resultados dos 18 estudos, no qual se verificou que a prevalência de idosos expostos a fragilidade teve uma ampla variação no percentual, sendo as mulheres em geral classificadas como mais frágeis do que os homens.

Dos estudos que analisaram o percentual de fragilidade apenas um teve o intuito de fazer um acompanhamento longitudinal realizando dois levantamentos, 1995/1996 e 2006 (6), com o mesmo instrumento de medida, no qual se pode verificar um aumento na proporção de idosos frágeis e pré-frágeis, tanto do sexo masculino quanto do sexo feminino com o passar dos anos.

Podem-se relacionar algumas das possíveis explicações para o entendimento da disparidade da prevalência da fragilidade descrito nos estudos:

a. Diferença no instrumento para avaliação da fragilidade, no qual se pode detectar cinco diferentes instrumentos utilizados e variações destes;

b. As diferenças na composição da amostra em relação à etnia e nacionalidade.

Há um desafio para os pesquisadores que é obter um instrumento que possa ser considerado um "padrão ouro" na mensuração do índice de fragilidade humana, a fim de que seja possível maior comparabilidade de resultados. Soma-se a esse desafio a necessidade de um instrumento que seja prático e válido em levantamentos populacionais que avaliem o grau de fragilidade em pessoas idosas em seu ambiente natural. Por questões logísticas, os questionários/entrevista continuam sendo utilizados com freqüência em populações mais velhas, embora 
esforços venham sendo realizados para o incremento na utilização de medidas de desempenho.

Apesar das limitações e variações metodológicas entre os estudos analisados, destaca-se o esforço de alguns pesquisadores que vêm conseguindo descrever a condição relacionada ao nível de fragilidade da população idosa. Em geral, as mulheres são mais frágeis do que os homens. As disparidades na prevalência de índices de fragilidade e pré-fragilidade devem ser minimizadas, com estímulo a padronização metodológica para a avaliação da fragilidade humana em populações idosas, ao desenvolvimento de estudos que discriminem as diferenças entre os cortes etários e as características regionais •

\section{REFERÊNCIAS}

1. Fried LP, Tangen CM, Walston J, Newman AB, Hirsch C, Gottdiener J, et al. Frailty in older adults: evidence for a phenotype. J Gerontol A Biol Sci Med Sci. 2001; 56(3):M146-56.

2. Rockwood K, Andrew M, Mitnitski A. A comparison of two approaches to measuring frailty in elderly people. J Gerontol A Biol Sci Med Sci. 2007; 62(7):738-43.

3. Ensrud KE, Ewing SK, Taylor BC, Fink HA, Stone KL, Cauley JA, et al. Frailty and risk of falls, fracture, and mortality in older women: the study of osteoporotic fractures. J Gerontol A Biol Sci Med Sci. 2007; 62(7):744-51.

4. Buchman AS, Wilson RS, Bienias JL, Bennett DA. Change in frailty and risk of death in older persons. Exp Aging Res. 2009; 35(1):61-82.

5. Klein BE, Klein R, Knudtson MD, Lee KE. Frailty, morbidity and survival. Arch Gerontol Geriatr. 2005; 41(2):141-9.

6. Ottenbacher KJ, Graham JE, Al Snih S, Raji M, Samper-Ternent R, Ostir GV, et al. Mexican Americans and frailty: findings from the Hispanic established populations epidemiologic studies of the elderly. Am J Public Health. 2009; 99(4):673-9.

7. Ensrud KE, Ewing SK, Taylor BC, Fink HA, Cawthon PM, Stone KL, et al. Comparison of 2 frailty indexes for prediction of falls, disability, fractures, and death in older women. Arch Intern Med. 2008; 168(4):382-9.

8. Bandeen-Roche K, Xue QL, Ferrucci L, Walston J, Guralnik JM, Chaves P, et al. Phenotype of frailty: characterization in the women's health and aging studies. J Gerontol A Biol Sci Med Sci. 2006; 61(3):262-6.

9. Cigolle CT, Ofstedal MB, Tian Z, Blaum CS. Comparing models of frailty: the Health and Retirement Study. J Am Geriatr Soc. 2009; 57(5):830-9.

10. Espinoza SE, Hazuda HP. Frailty in older Mexican-American and European-American adults: is there an ethnic disparity? J Am Geriatr Soc. 2008; 56(9):1744-9.

11. Hirsch C, Anderson ML, Newman A, Kop W, Jackson S, Gottdiener J, et al. The association of race with frailty: the cardiovascular health study. Ann Epidemiol. 2006; 16(7):545-53.

12. Santos-Eggimann B, Cuénoud P, Spagnoli J, Junod J. Prevalence of frailty in middle-aged and older community-dwelling Europeans living in 10 countries. J Gerontol A Biol Sci Med Sci. 2009; 64(6):675-81.

13. Brown M, Sinacore DR, Binder EF, Kohrt WM. Physical and performance measures for the identification of mild to moderate frailty. J Gerontol A Biol Sci Med Sci. 2000; 5(6):M350-5.

14. Villareal DT, Banks M, Siener C, Sinacore DR, Klein S. Physical frailty and body composition in obese elderly men and women. Obes Res. 2004; 12(6):913-20. 
15. Kirby SE, Coleman PG, Daley D. Spirituality and well-being in frail and nonfrail older adults. J Gerontol B Psychol Sci Soc Sci. 2004; 59(3):P123-9.

16. Mitnitski AB, Graham JE, Mogilner AJ, Rockwood K. Frailty, fitness and late-life mortality in relation to chronological and biological age. BMC Geriatr. 2002; 2:1.

17. Gu D, Dupre ME, Sautter J, Zhu H, Liu Y, Yi Z. Frailty and mortality among Chinese at advanced ages. J Gerontol B Psychol Sci Soc Sci. 2009; 64(2):279-89.

18. Dupre ME, Gu D, Warner DF, Yi Z. Frailty and type of death among older adults in China: prospective cohort study. BMJ. 2009; 338:b1175.

19. Mitnitski AB, Song X, Rockwood K. The estimation of relative fitness and frailty in communitydwelling older adults using self-report data. J Gerontol A Biol Sci Med Sci. 2004; 59(6):M627-32.

20. Song X, Mitnitski A, MacKnight C, Rockwood K. Assessment of individual risk of death using self-report data: an artificial neural network compared with a frailty index. J Am Geriatr Soc. 2004; 52(7):1180-4.

21. Song X, MacKnight C, Latta R, Mitnitski AB, Rockwood K. Frailty and survival of rural and urban seniors: results from the Canadian Study of Health and Aging. Aging Clin Exp Res. 2007; 19(2): $145-53$

22. Goggins WB, Woo J, Sham A, Ho SC. Frailty index as a measure of biological age in a Chinese population. J Gerontol A Biol Sci Med Sci. 2005; 60(8):1046-51.

23. Woo J, Goggins W, Sham A, Ho SC. Social determinants of frailty. Gerontology. 2005; 51(6):402-8.

24. Rockwood K, Howlett SE, MacKnight C, Beattie BL, Bergman H, Hébert R, et al. Prevalence, attributes, and outcomes of fitness and frailty in community-dwelling older adults: report from the Canadian study of health and aging. J Gerontol A Biol Sci Med Sci. 2004; 59(12):1310-7.

25. Puts MT, Lips P, Deeg DJ. Sex differences in the risk of frailty for mortality independent of disability and chronic diseases. J Am Geriatr Soc. 2005; 53(1):40-7.

26. Blaum CS, Xue QL, Michelon E, Semba RD, Fried LP. The association between obesity and the frailty syndrome in older women: the Women's Health and Aging Studies. J Am Geriatr Soc. 2005; 53(6):927-34.

27. Ottenbacher KJ, Ostir GV, Peek MK, Snih SA, Raji MA, Markides KS. Frailty in older Mexican Americans. J Am Geriatr Soc. 2005; 53(9):1524-31.

28. Woods NF, LaCroix AZ, Gray SL, Aragaki A, Cochrane BB, Brunner RL, et al. Frailty: emergence and consequences in women aged 65 and older in the Women's Health Initiative Observational Study. J Am Geriatr Soc. 2005; 53(8):1321-30.

29. Bartali B, Frongillo EA, Bandinelli S, Lauretani F, Semba RD, Fried LP, et al. Low nutrient intake is an essential component of frailty in older persons. J Gerontol A Biol Sci Med Sci. 2006; 61(6):589-93.

30. Cesari M, Leeuwenburgh C, Lauretani F, Onder G, Bandinelli S, Maraldi C, et al. Frailty syndrome and skeletal muscle: results from the Invecchiare in Chianti study. Am J Clin Nutr. 2006; 83(5):1142-8.

31. Cawthon PM, Marshall LM, Michael Y, Dam TT, Ensrud KE, Barrett-Connor E, et al. Frailty in older men: prevalence, progression, and relationship with mortality. J Am Geriatr Soc. 2007; 55(8):1216-23.

32. Andrew MK, Mitnitski AB, Rockwood K. Social vulnerability, frailty and mortality in elderly people. PLoS One. 2008; 3(5):e2232.

33. Avila-Funes JA, Helmer C, Amieva H, Barberger-Gateau P, Le Goff M, Ritchie K, et al. Frailty among community-dwelling elderly people in France: the three-city study. J Gerontol A Biol Sci Med Sci. 2008; 63(10):1089-1096.

34. Blyth FM, Rochat S, Cumming RG, Creasey H, Handelsman DJ, Le Couteur DG, et al. Pain, frailty and comorbidity on older men: the CHAMP study. Pain. 2008; 140(1):224-30.

35. Masel MC, Graham JE, Reistetter TA, Markides KS, Ottenbacher KJ. Frailty and health related quality of life in older Mexican Americans. Health Qual Life Outcomes. 2009; 7:70. 